\title{
Hermite Polynomial Normal Transformation for Structural Reliability Analysis
}

\author{
Jinsheng Wang ${ }^{\mathrm{a}}$, Muhannad Aldosary ${ }^{\mathrm{a}, \mathrm{b}}$, Song Cen ${ }^{\mathrm{c}}$, and Chenfeng Li ${ }^{* a, \mathrm{~d}}$ \\ aZienkiewicz Centre for Computational Engineering, College of Engineering, Swansea \\ University Bay Campus, Swansea SA1 8EN, United Kingdom \\ ${ }^{\mathrm{b}}$ Dams and Water Resources Engineering, University of Anbar, Iraq \\ ${ }^{\mathrm{c} S c h o o l ~ o f ~ A e r o s p a c e ~ E n g i n e e r i n g, ~ T s i n g h u a ~ U n i v e r s i t y, ~ B e i j i n g ~ 100084, ~ C h i n a ~}$ \\ dEnergy Safety Research Institute, College of Engineering, Swansea University Bay Campus, \\ Swansea SA1 8EN, United Kingdom
}

\begin{abstract}
Purpose: Normal transformation is often required in structural reliability analysis to convert the non-normal random variables into independent standard normal variables. The existing normal transformation techniques, e.g. Rosenblatt transformation and Nataf transformation, usually require the joint probability density function (PDF) and/or marginal PDFs of nonnormal random variables. In practical problems, however, the joint PDF and marginal PDFs are often unknown due to the lack of data while the statistical information is much easier to be expressed in terms of statistical moments and correlation coefficients. The study aims to address this issue, by presenting an alternative normal transformation method that does not require PDFs of the input random variables.
\end{abstract}

Design/methodology/approach: The new approach, namely the Hermite polynomial normal transformation, expresses the normal transformation function in terms of Hermite polynomials and it works with both uncorrelated and correlated random variables. Its application in structural reliability analysis using different methods is thoroughly investigated via a number of carefully designed comparison studies.

Findings: Comprehensive comparisons are conducted to examine the performance of the proposed Hermite polynomial normal transformation scheme. The results show that the presented approach has comparable accuracy to previous methods and can be obtained in closed-form. Moreover, the new scheme only requires the first four statistical moments and/or the correlation coefficients between random variables, which greatly widen the applicability of normal transformations in practical problems.

Originality/value: This study interprets the classical polynomial normal transformation method in terms of Hermite polynomials, namely Hermite polynomial normal transformation, to convert uncorrelated/correlated random variables into standard normal random variables. The new scheme only requires the first four statistical moments to operate, making it particularly suitable for problems that are constraint by limited data. Besides, the extension to correlated cases can easily be achieved with the introducing of the Hermite polynomials. Compared to existing methods, the new scheme is cheap to compute and delivers comparable accuracy.

Keywords: Structural reliability analysis, Polynomial normal transformation, Hermite polynomials, Statistical moments

\footnotetext{
${ }^{*}$ Corresponding author: c.f.li@swansea.ac.uk
} 


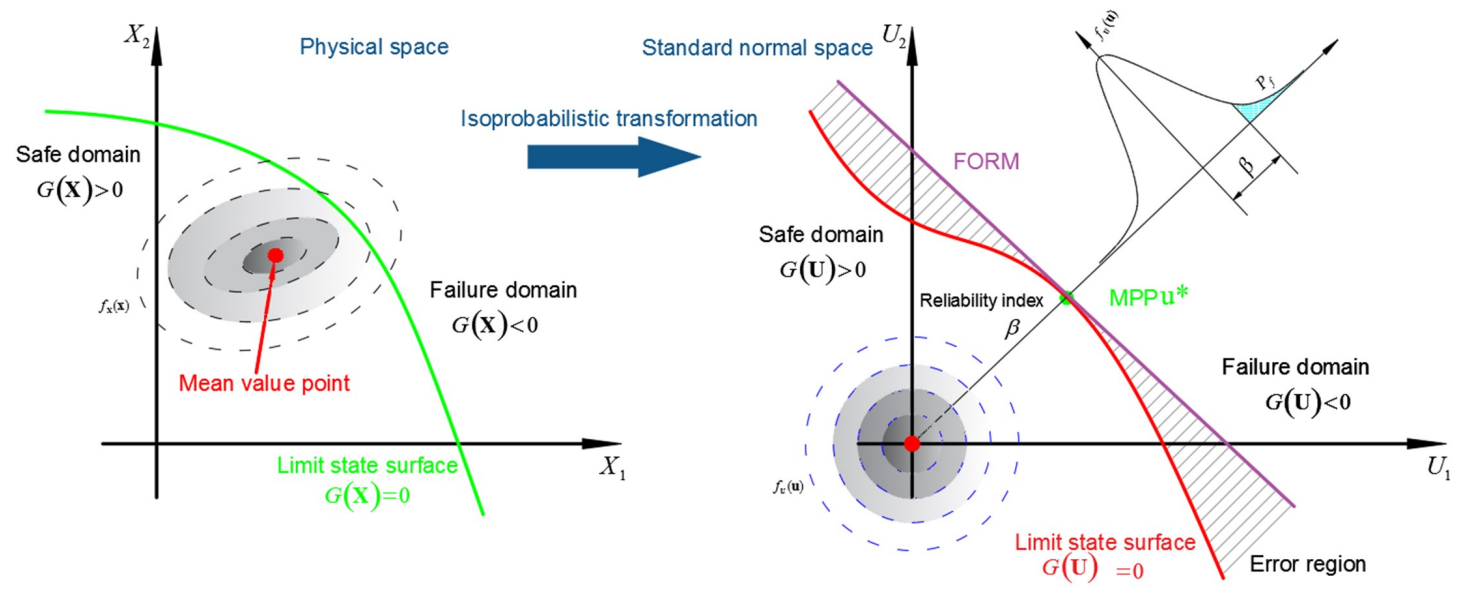

Figure 1: Transformation from physical space to standard normal space (Hu et al., 2019).

\section{Introduction}

Structural reliability analysis aims to determine the probability of failure for a structural system, and it is the theoretical foundation for the associated risk assessment and reliability-based optimal design. In engineering practice, the uncertainties can arise from loading conditions, material properties, environmental factors and modelling errors, which are often formulated as a random vector $\mathbf{X}=\left[X_{1}, X_{2}, \ldots, X_{n}\right]^{T}$ defined in the physical space. The probability of failure is expressed as a multidimensional integral:

$$
P_{f}=\operatorname{Prob}[g(\boldsymbol{x}) \leq 0]=\int_{g(\boldsymbol{x}) \leq 0} f_{\mathbf{X}}(\boldsymbol{x}) d \boldsymbol{x}
$$

where $f_{\mathbf{X}}(\boldsymbol{x})$ is the joint probability density function (PDF) of random vector $\mathbf{X}, g(\boldsymbol{x})$ is the performance function that $g(\boldsymbol{x}) \leq 0$ denotes the failure domain and $g(\boldsymbol{x})>0$ the safe domain.

Despite the simple definition in Eq. (1), the accurate evaluation of failure probability is often very difficult through direct integration, in that for practical engineering problems the dimension of integral is usually high and the performance function is of complicated shape. Moreover, direct integration is unfeasible when the joint PDF of random variables is unknown a priori. The challenge of accurately computing this integral has led to the development of various approximation methods, such as the firstorder reliability method (FORM) (Hasofer and Lind, 1974; Shinozuka, 1983), second-order reliability method (SORM) (Breitung, 1984; Der Kiureghian et al., 1987), Monte Carlo simulation (MCS) (Naess et al., 2012), importance sampling methods (Au and Beck, 2003), directional simulation methods (Nie and Ellingwood, 2000), subset simulation (Au and Beck, 2001), moment-based methods (Zhao and Ono, 2001) and various surrogate-based approaches (Aldosary et al., 2018; Zhang and Xiao, 2019; Yangtian Li, 2020). It is almost always desirable to conduct the aforementioned methods in the standard normal space, thanks for its simplicity. Therefore, when non-normal random variables are present in the physical space, normal transformation techniques are applied to convert the non-normal random variable into standard normal ones before performing the reliability analysis. A schematic illustration for normal transformation is shown in Fig. 1. The Rosenblatt transformation (Rosenblatt, 1952; Hohenbichler and Rackwitz, 1981) and the Nataf transformation (Der Kiureghian and Liu, 1986) are two most widely used approaches for this purpose. The former one requires the cumulative distribution function (CDF) or joint $\mathrm{PDF}$ of random variables in the physical space, and the later one requires the marginal distribution function of the random variables. In engineering practice, however, the CDF/PDF of some random variables may be unknown due to the lack of data, whereas the probabilistic properties of these random variables is easier to be expressed in terms of statistical moments and correlation coefficients (Zhao and Ono, 2000). In such cases, a strict failure probability evaluation using the conventional transformation techniques is unfeasible unless additional effort is made to fit an appropriate distribution.

This paper presents an alternative approach, namely the polynomial normal transformation, to 


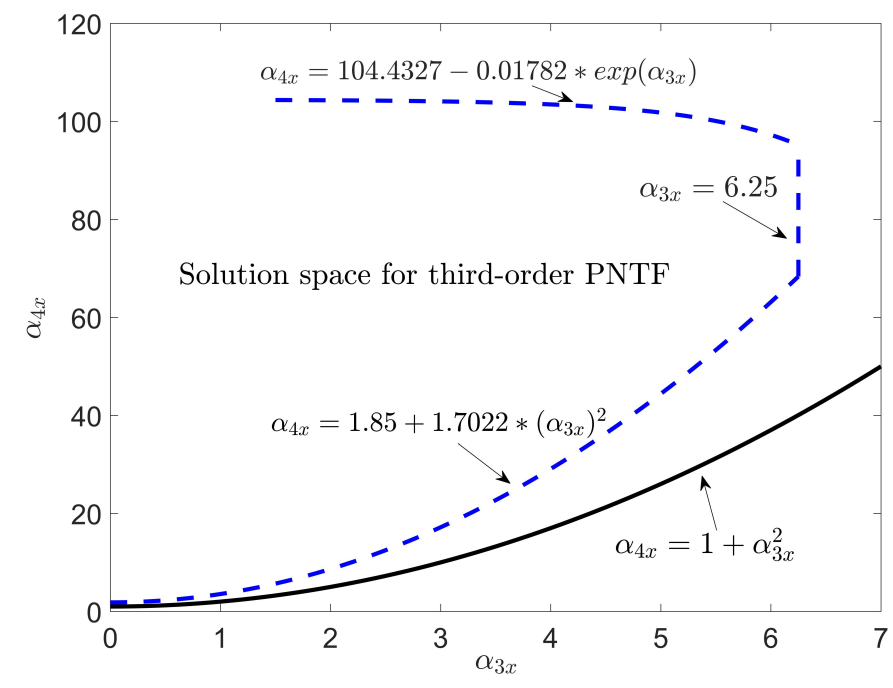

Figure 2: The solution condition for the third-order PNTF (Chen and Tung, 2003).

convert non-normal random variables in the physical space into standard normal random variables. In this approach, the normal transformation and its inverse can be performed by using only the statistical moments and correlation coefficients between random variables in the physical space. As a result, the reliability analysis can be carried out without the $\mathrm{CDF} / \mathrm{PDF}$ of input random variables, and hence greatly widens the applicability. The rest of the paper is organised as follows. $\S 2$ briefly recaps the existing polynomial normal transformation functions (PNTF) and the common approaches to determine the polynomial coefficients. $\S 3$ presents the PNTF in terms of Hermite polynomials, which works for correlated input random variables and offers an explicit solution when the marginal PDFs of random inputs are known. In $\S 4$, the Hermite PNTF is combined with standard reliability analysis methods, e.g. MCS and FORM/SORM, to demonstrate and examine its performance. Finally, concluding remarks are summarised in $\S 5$.

\section{A brief overview of polynomial normal transformation functions}

Normal transformation is often desired in probabilistic analysis especially for problems involving multivariate non-normal random variables. Rosenblatt and Nataf transformations are the two most commonly used approaches to convert non-normal random variables to normal ones. However, as discussed earlier, these two classic methods cannot be employed for cases where the CDF/PDF of input random variables are unknown due to lack of data. Indeed, it is often easier to quantify practical random variables in terms of their statistical moments and correlation coefficients. In these cases, the polynomial normal transformation, also known as the power method, provides a promising alternative to conduct the non-normal to normal transformation. The polynomial normal transformation has been used in many literatures (Zhao and Ono, 2000; Zhao and Lu, 2007; Tung et al., 2019; Zhang et al., 2019), where the non-normal random variables are expressed as polynomial functions of the normal ones, referred to as polynomial normal transformation functions (PNTF):

$$
\frac{X_{i}-\mu_{x_{i}}}{\sigma_{x_{i}}}=X_{S i} \simeq \sum_{k=0}^{P} a_{k i} Z_{i}^{k}
$$

where $X_{i}$ is the $i$-th entry in the random vector $\mathbf{X}, \mu_{x_{i}}$ denotes its mean value, $\sigma_{x_{i}}$ denotes its variance, $X_{S i}$ is the standardized variable of $X_{i}$ with zero mean and unit variance, $a_{k i}, k=0,1,2, \ldots, P$ are the unknown polynomial coefficients to be determined, $Z_{i}$ is the $i$-th entry of the independent standardized normal vector $\boldsymbol{Z}$. 
A third-order PNTF was first proposed by Fleishman (1978) to generate non-normal random variables with specified moments, and it is expressed as:

$$
X_{S i}=a_{0 i}+a_{1 i} Z_{i}+a_{2 i} Z_{i}^{2}+a_{3 i} Z_{i}^{3}
$$

where the polynomial coefficients $a_{k i}, k=0,1,2,3$ are determined by equalling the first four central moments of both sides of Eq. (3):

$$
\begin{gathered}
0=a_{0 i}+a_{2 i} \\
1=a_{1 i}^{2}+6 a_{1 i} a_{3 i}+2 a_{2 i}^{2}+15 a_{3 i}^{2} \\
\alpha_{3 x_{i}}=2 a_{2 i}\left(a_{1 i}^{2}+24 a_{1 i} a_{3 i}+105 a_{3 i}^{2}+2\right) \\
\alpha_{4 x_{i}}-3=24\left[a_{1 i} a_{3 i}+a_{2 i}^{2}\left(1+a_{1 i}^{2}+28 a_{1 i} a_{3 i}\right)+a_{3 i}^{2}\left(12+48 a_{1 i} a_{3 i}+141 a_{2 i}^{2}+225 a_{3 i}^{2}\right)\right]
\end{gathered}
$$

where $\alpha_{3 x_{i}}$ and $\alpha_{4 x_{i}}$ are skewness and kurtosis of $X_{i}$, respectively. This approach to determining the polynomial coefficients $a_{k i}$ is known as the product-moment method. Its accuracy and monotonicity range were investigated in (Chen and Tung, 2003). Once the coefficients $a_{k i}$ are obtained, the non-normal random variables can be simulated by substituting normal samples into Eq. (3). The solution for the above product-moment equations (4-7) depends on the values of skewness and kurtosis (Fleishman, 1978). When skewness is less than 2.5 and kurtosis less than 9 , the solution exists if the following condition holds:

$$
\alpha_{4 X}>1.58857 \alpha_{3 X}^{2}+1.8683
$$

For skewness less than 10 and kurtosis less than 120, the solution condition for Eqs. (4-7) was studied in (Chen and Tung, 2003) and is depicted in Fig. 2, where the relation $\alpha_{4 X} \geq 1+\alpha_{3 X}^{2}$ holds for a feasible distribution (Stuart et al., 1994).

Tadikamalla (1980) presented five alternative methods for generating non-normal distributions and compared them with the third-order PNTF in terms of the efficiency, implementation and generality. The third-order PNTF was found to be the easiest for implementation and with the highest efficiency. In addition to the third-order polynomial transformation in Eq. (3), a closed form second-order PNTF was derived in (Zhao and Ono, 2000) using the information of the first three moments. Some other formulations for normal transformation have also been proposed over the years, among which the secondorder Fisher-Cornish expansion is given in an explicit form as (Chen and Tung, 2003; Zhao and Lu, 2007):

$$
X_{S}=-a_{1}+\left(1-3 a_{2}\right) Z+a_{1} Z^{2}+a_{2} Z^{3}
$$

where $X_{S}=\frac{X-\mu_{x}}{\sigma_{x}}, a_{1}=\frac{\alpha_{3 x}}{6}, a_{2}=\frac{\alpha_{4 x}-3}{24}$. The above closed-form PNTF is convenient to use. However, as pointed out in (Zhao and Lu, 2007), the first four moments of the right-hand side of Eq. (9) are not equal to those of the left-hand side, and large errors may occur when applying the transformation.

An improved version to Eq. (9) was presented in (Winterstein and Bjerager, 1987):

$$
X_{S}=-k b_{1}+k\left(1-3 b_{2}\right) Z+k b_{1} Z^{2}+k b_{2} Z^{3}
$$

where

$$
\begin{gathered}
b_{1}=\frac{\alpha_{3 x}}{4+2 \sqrt{1+1.5\left(\alpha_{4 x}-3\right)}} \\
b_{2}=\frac{\sqrt{1+1.5\left(\alpha_{4 x}-3\right)}-1}{18} \\
k=\frac{1}{\sqrt{1+2 b_{1}^{2}+6 b_{2}^{2}}}
\end{gathered}
$$

The above formula improves the accuracy of the Fisher-Cornish expansion while retaining explicit.

A further improvement was proposed in (Zhao and Lu, 2007):

$$
X_{S}=-l_{1}+k_{1} Z+l_{1} Z^{2}+k_{2} Z^{3}
$$


where the polynomial coefficients were derived from trial and error using a large amount of data as:

$$
\begin{gathered}
l_{1}=\frac{\alpha_{3 x}}{6\left(1+6 l_{2}\right)}, l_{2}=\frac{1}{36}\left(\sqrt{6 \alpha_{4 x}-8 \alpha_{3 x}^{2}-14}-2\right) \\
k_{1}=\frac{1-3 l_{2}}{1+l_{1}^{2}-l_{2}^{2}}, k_{2}=\frac{l_{2}}{1+l_{1}^{2}+12 l_{2}^{2}}
\end{gathered}
$$

The following condition must hold for the validity of Eq. (14):

$$
\alpha_{4 x} \geq\left(7+4 \alpha_{3 x}^{2}\right) / 3
$$

According to the study of (Zhao and Lu, 2007), the range expressed in Eq. (17) can generally meet the common engineering use. An extension of Eq. (14) was recently presented in (Zhao et al., 2019).

All aforementioned polynomial transformation functions are limited to independent random variables. For the simulation of multivariate non-normal random variables with specified moments and correlation matrix, further improvement have been reported in (Vale and Maurelli, 1983; Headrick and Sawilowsky, 1999).

The application of PNTF usually involves matching moments up to the fourth-order, and results of sufficient accuracy can generally be obtained. One way to further improve the approximation accuracy generated by moment-matching is to account for higher-order moments. In this regard, a PNTF of order five was proposed in (Headrick, 2002), and it offers additional control over the fifth and sixth moments to approximate some distributions that are difficult for the lower-order approach. Its implementation, however, requires rather tedious mathematical computation to determine the polynomial coefficients. To simplify the coefficient estimation, L-moments were used to establish the fifth-order PNTF and the corresponding analytical expressions were derived in (Headrick, 2011). Along this line of development, a PNTF of order 19 was proposed in (Xiao, 2015) where the polynomial coefficients are determined using the probability weighted moments matching method or the percentile matching method. Based on a similar idea, a 9-th order polynomial transformation was used with a quasi-Monte Carlo method to solve for the probabilistic optimal power flow problem (Zou and Xiao, 2014).

It is noted that the third-order PNTF has recently been further employed to construct a flexible probability distribution for a random variable only with the first four moments in (Zhao et al., 2018b), whereas the application of third-order PNTF to establish a multivariate distribution model has previously been reported in (Chen and Tung, 2003). For more information, it is referred to (Chen and Tung, 2003; Headrick and Kowalchuk, 2007; Zhao et al., 2018b) and references therein.

\section{PNTF in terms of Hermite polynomials}

As Hermite polynomials are orthogonal with each other weighted by the PDF of standard normal distribution (Andrews et al., 1999; Xiu and Karniadakis, 2002), it is attractive to use Hermite polynomials as the PNTF to link non-normal random variables to normal ones:

$$
X_{S i}=\sum_{k=0}^{P} b_{k i} H_{k}\left(Z_{i}\right)
$$

where $X_{S i}$ is the standardized non-normal random variable, $k$ is the order of expansion, $b_{k i}$ is the $k$-th unknown coefficients to be determined, and $H_{k}(\bullet)$ is the $k$-th order Hermite polynomial. For a finite order expansion, it is trivial to build the connection between the Hermite expansion in Eq. (18) and the power series in Eq. (2). For the sake of simplicity, a third order expansion is considered here:

$$
X_{S i}=\sum_{k=0}^{3} b_{k i} H_{k}\left(Z_{i}\right)=b_{0 i}+b_{1 i} Z_{i}+b_{2 i}\left(Z_{i}^{2}-1\right)+b_{3 i}\left(Z_{i}^{3}-3 Z_{i}\right)
$$


Comparing Eq. (19) with Eq. (3), the relationships between the expansion coefficients in these two equations can be obtained as:

$$
\begin{aligned}
& b_{0 i}=a_{0 i}+a_{2 i} ; b_{1 i}=a_{1 i}+3 a_{3 i} ; \\
& b_{2 i}=a_{2 i} ; b_{3 i}=a_{3 i} ;
\end{aligned}
$$

Following Eq. (19), the moments of $X_{S i}$ can be calculated as:

$$
E\left[X_{S i}^{m}\right]=E\left[\left\{\sum_{k=0}^{3} b_{k i} H_{k}\left(Z_{i}\right)\right\}^{m}\right]=E\left[\left\{b_{0 i}+b_{1 i} Z_{i}+b_{2 i}\left(Z_{i}^{2}-1\right)+b_{3 i}\left(Z_{i}^{3}-3 Z_{i}\right)\right\}^{m}\right]
$$

Based on the moment matching method, the following set of equations can be established for solving the unknown coefficients when $m=4$ :

$$
\begin{aligned}
& 0=b_{0 i} \\
& 1=b_{1 i}^{2}+2 b_{2 i}^{2}+6 b_{3 i}^{2} \\
& \alpha_{3 x_{i}}=2 b_{2 i}\left(2+b_{1 i}^{2}+18 b_{1 i} b_{3 i}+42 b_{3 i}^{2}\right) \\
& \alpha_{4 x_{i}}=15+288 b_{1 i} b_{3 i}+936 b_{3 i}^{2}-12 b_{1 i}^{4}-264 b_{1 i}^{3} b_{3 i}-864 b_{1 i}^{2} b_{3 i}^{2}-432 b_{1 i} b_{3 i}^{3}-2808 b_{3 i}^{4}
\end{aligned}
$$

then, the same set of equations can be derived as expressed in Eqs. (4-7) by substituting Eq. (20) into Eq. (22), which in a way establish the connection between the Hermite polynomial expansion in Eq. (18) and the power series in Eq. (2) by restricting $P=3$.

The benefits of expressing PNTF in terms of Hermite polynomials are explained in the following two subsections, from two different aspects: 1) simple and efficient calculation of the PNTF coefficients for random variables with specified distribution; and 2) convenience to cope with correlated non-normal random variables.

\subsection{Coefficients calculation for random variables with specified distributions}

Although the PNTF for normal transformation only requires the statistical moments of random variables, interesting properties can be observed for the Hermite PNTF applied to random variables with specified distributions. Unlike in Eq. (18) where the left-hand side is a standardized random variable, here the original random variable $X_{i}$ is directly expanded as a weighted summation of Hermite polynomials:

$$
X_{i}=\sum_{k=0}^{P} c_{k i} H_{k}\left(Z_{i}\right)
$$

Due to the orthogonality of Hermite polynomials and their moment properties, the following equation can be derived:

$$
E\left[X_{i} \cdot H_{k}\left(Z_{i}\right)\right]=c_{k i} E\left[\left(\sum_{k=0}^{P} H_{k}\left(Z_{i}\right)\right) H_{k}\left(Z_{i}\right)\right]=c_{k i} E\left[H_{k}^{2}\left(Z_{i}\right)\right]=c_{k i} k !
$$

The isoprobabilistic transformation between the non-normal random variable $X_{i}$ and the standard normal variable $Z_{i}$ is given as:

$$
F_{X}\left(X_{i}\right)=\Phi\left(Z_{i}\right) \rightarrow X_{i}=F_{X}^{-1}\left(\Phi\left(Z_{i}\right)\right)
$$

where $F_{X}(\bullet)$ and $\Phi(\bullet)$, respectively, are the CDF of non-normal random variable and standard normal random variable.

Substituting Eq. (25) into Eq. (24) yields:

$$
E\left[X_{i} \cdot H_{k}\left(Z_{i}\right)\right]=\int_{R} F_{X}^{-1}\left(\Phi\left(Z_{i}\right)\right) H_{k}\left(Z_{i}\right) \phi\left(Z_{i}\right) d Z_{i}=c_{k i} k !
$$




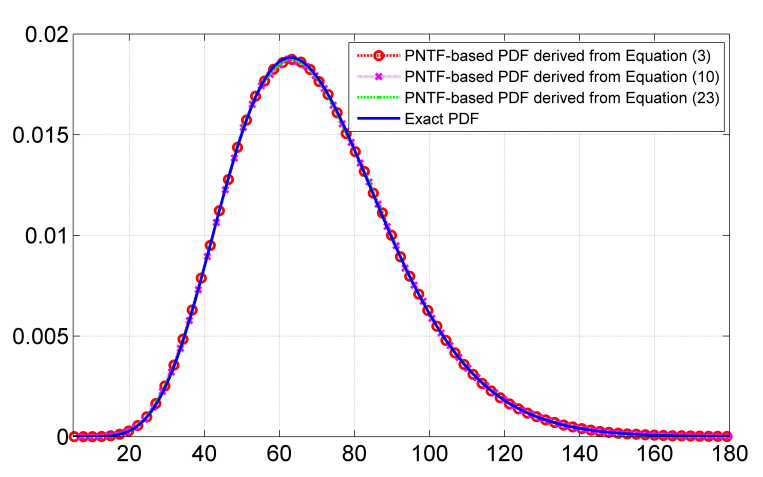

(a)

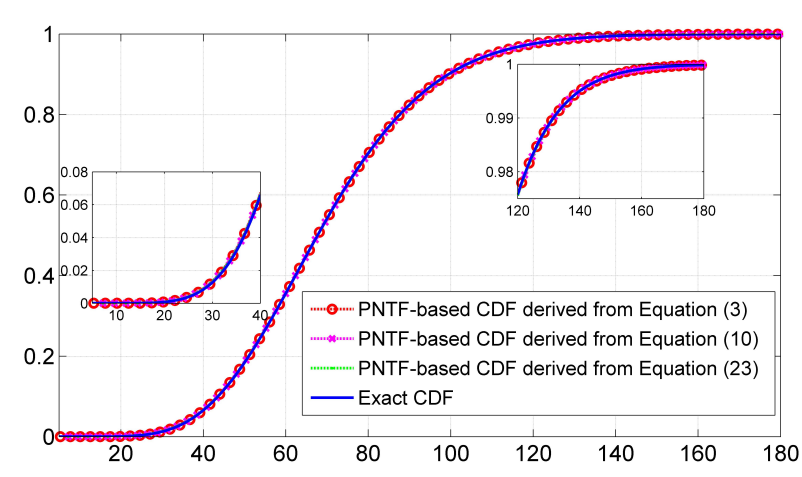

(b)

Figure 3: Approximation of the Gamma distribution using third-order PNTF derived from different methods: (a) PDF; (b) CDF.

where $\phi(\bullet)$ is the PDF of a standard normal random variable. Finally, the polynomial coefficients can easily be calculated as a one-dimensional integral for any order of expansion:

$$
c_{k i}=\frac{1}{k !} \int_{R} F_{X}^{-1}\left(\Phi\left(Z_{i}\right)\right) H_{k}\left(Z_{i}\right) \phi\left(Z_{i}\right) d Z_{i}
$$

For example, assuming that $X_{1}$ follows Gamma distribution with the shape parameter of 10 and scale parameter of 7, a third-order PNTF in the form of Eq. (23) is used to represent $X_{1}$. With the aid of Eq. (27), the four polynomial coefficients can readily be calculated as: $c_{01}=70, c_{11}=21.8928$, $c_{21}=2.3111$ and $c_{31}=0.0634$. Therefore, the random variable $X_{1}$ can be expressed in terms of Hermite polynomials as:

$$
X_{1}=70+21.8928 Z_{1}+2.3111\left(Z_{1}^{2}-1\right)+0.0634\left(Z_{1}^{3}-3 Z_{1}\right)
$$

By generating a large set of realizations of the standard normal random variable $Z_{1}$, the PNTF approximation of the PDF and CDF of Gamma random variable $X_{1}$ are illustrated in Fig. 3. The PNTF-based PDF/CDFs derived from moment matching methods mentioned in Section 2, i.e. Eq. (3) and Eq. (14), are also shown in Fig. 3 for comparison purpose. It is observed that all the PNTF-based $\mathrm{PDF} / \mathrm{CDFs}$ exhibit a close agreement with each other, and match with the exact ones very well in the whole domain, even in the tail part of the distribution. Therefore, both the moment matching methods and Eq. (27) can be used to calculate the coefficients for establishing the PNTF-based transformation.

\subsection{Extension to correlated random variables}

Another advantage for expressing the PTNF as Hermite polynomials is its convenience to establish the relationship between the correlation coefficients in the original space and that in the standard normal space, which leads to the normal transformation that links correlated non-normal random variables to independent standard normal ones. Specifically, given two correlated random variables $X_{i}$ and $X_{j}$, their corresponding standardized variables $X_{S i}, X_{S j}$ can be expressed as:

$$
\begin{gathered}
\frac{X_{i}-\mu_{x_{i}}}{\sigma_{x_{i}}}=X_{S i}=\sum_{k=0}^{P} b_{k i} H_{k}\left(Z_{i}\right) \\
\frac{X_{j}-\mu_{x_{j}}}{\sigma_{x_{j}}}=X_{S j}=\sum_{k=0}^{P} b_{k j} H_{k}\left(Z_{j}\right)
\end{gathered}
$$


where $X_{i}$ and $X_{j}$ are the desired non-normal random variables with correlation coefficient of $\rho_{x_{i} x_{j}}, X_{S i}$, $X_{S j}$ are the standardized variables of $X_{i}$ and $X_{j}$ with zero mean and unit variance, $Z_{i}$ and $Z_{j}$ are the standard normal random variables with the correlation coefficient $\rho_{z_{i} z_{j}}$. Then according to the following property of Hermite polynomials (Buet-Golfouse, 2015):

$$
\operatorname{Cov}\left[H_{m}\left(Z_{i}\right), H_{n}\left(Z_{j}\right)\right]=\left\{\begin{array}{cl}
0 & \text { for } \mathrm{n} \neq \mathrm{m} \\
n ! \rho_{z_{i} z_{j}}^{n} & \text { for } \mathrm{n}=\mathrm{m}>0
\end{array}\right.
$$

the following equation can be derived:

$$
\operatorname{Cov}\left(X_{S i}, X_{S j}\right)=\rho_{X_{S i} X_{S j}} \sigma_{X_{S i}} \sigma_{X_{S j}}=\rho_{x_{i} x_{j}}=\sum_{k=1}^{P} b_{k i} b_{k j} k ! \rho_{z_{i} z_{j}}^{k}
$$

Thus, the relationship between the correlation coefficient in the original space $\rho_{x_{i} x_{j}}$ and the correlation coefficient in the standard normal space $\rho_{z_{i} z_{j}}$ is established, which is formulated as a polynomial function of $\rho_{z_{i} z_{j}}$ up to degree $P$. For practical application purpose, the expansion up to 3-order (i.e. $P=3)$ is generally employed. According to the relationship between the parameter vectors $\mathbf{a}$ and $\mathbf{b}$ expressed in Eq. (20), Eq. (32) can also be expressed in terms of the parameter vector a:

$$
6 \alpha_{3 i} \alpha_{3 j} \rho_{z_{i} z_{j}}^{3}+2 a_{2 i} a_{2 j} \rho_{z_{i} z_{j}}^{2}+\left(\alpha_{1 i}+3 \alpha_{3 i}\right)\left(\alpha_{1 j}+3 \alpha_{3 j}\right) \rho_{z_{i} z_{j}}-\rho_{x_{i} x_{j}}=0
$$

The correlation coefficient in standard normal space $\rho_{z_{i} z_{j}}$ can then be determined by solving Eq. (32) or Eq. (33). In order to satisfy the definition of correlation coefficient, the valid solution is restricted by the following conditions (Tung et al., 2019; Xiao, 2017):

$$
\begin{aligned}
& -1 \leq \rho_{z_{i} z_{j}} \leq 1 ; \\
& \rho_{z_{i} z_{j}} \cdot \rho_{x_{i} x_{j}} \geq 0 ;
\end{aligned}
$$

Therefore, following the above derivations, any pair of statistically dependent random variables with the first four moments and correlation coefficients available can be transformed into two correlated standard normal variables, whose correlation coefficients can easily be determine from Eq. (32) or Eq. (33).

The aforementioned transformation procedure can readily be generalized to $n$ variables, whose joint $\mathrm{CDF} /$ marginal PDFs are unknown and the probabilistic characteristics are expressed as the first four moments and correlation coefficients. Consider a $n$ dimensional random vector $\mathbf{X}=\left[X_{1}, X_{2}, \cdots, X_{n}\right]^{T}$ with the probabilistic information defined by the first four moments $\left(\mu_{x_{i}}, \sigma_{x_{i}}, \alpha_{3 x_{i}}, \alpha_{4 x_{i}}\right)$ and the correlation matrix:

$$
\mathbf{R}_{\mathbf{X}}=\left(\begin{array}{cccc}
1 & \rho_{x_{1} x_{2}} & \cdots & \rho_{x_{1} x_{n}} \\
\rho_{x_{2} x_{1}} & 1 & \cdots & \rho_{x_{2} x_{n}} \\
\vdots & \vdots & \ddots & \vdots \\
\rho_{x_{n} x_{1}} & \rho_{x_{n} x_{2}} & \cdots & 1
\end{array}\right)
$$

Then, each pair of the random variables in $\mathbf{X}$ can be transformed into a pair of correlated standard normal variable in $\mathbf{Z}=\left[Z_{1}, Z_{2}, \cdots, Z_{n}\right]^{T}$ using Eqs. (29-30). The elements of the correlation matrix in the standard normal space can be constructed by solving Eq. (32) or Eq. (33) for each pair of random variables and is expressed as:

$$
\mathbf{R}_{\mathbf{Z}}=\left(\begin{array}{cccc}
1 & \rho_{z_{1} z_{2}} & \cdots & \rho_{z_{1} z_{n}} \\
\rho_{z_{2} z_{1}} & 1 & \cdots & \rho_{z_{2} z_{n}} \\
\vdots & \vdots & \ddots & \vdots \\
\rho_{z_{n} z_{1}} & \rho_{z_{n} z_{2}} & \cdots & 1
\end{array}\right)
$$

Given a positive definite correlation matrix $\mathbf{R}_{\mathbf{Z}}$ of statistically dependent standard normal vector $\mathbf{Z}=\left[Z_{1}, Z_{2}, \cdots, Z_{n}\right]^{T}$, an independent standard normal random vector $\mathbf{U}=\left[U_{1}, U_{2}, \cdots, U_{n}\right]^{T}$ can be formulated as:

$$
\mathbf{U}=\mathbf{L}^{-1} \mathbf{Z}
$$


where $\mathbf{L}$ is a lower-triangular matrix obtained from Cholesky decomposition of the correlation matrix $\mathbf{R}_{\mathbf{Z}}$ such that $\mathbf{R}_{\mathbf{Z}}=\mathbf{L L}^{\mathbf{T}}$. The lower-triangular matrix $\mathbf{L}$ is expressed as:

$$
\mathbf{L}=\left(\begin{array}{cccc}
l_{11} & 0 & \cdots & 0 \\
l_{21} & l_{22} & \cdots & 0 \\
\vdots & \vdots & \ddots & \vdots \\
l_{n 1} & l_{n 2} & \cdots & l_{n n}
\end{array}\right)
$$

From Eq. (37), the vector $\mathbf{Z}$ can be expressed in terms of an independent standard normal vector $\mathbf{U}$ as:

$$
\mathbf{Z}=\mathbf{L} \mathbf{U}
$$

where the element of $\mathbf{Z}$ can be expressed as:

$$
Z_{i}=\sum_{m=1}^{i} l_{i m} U_{m},(i=1,2, \ldots, n)
$$

By substituting Eq. (40) into Eq. (29), the PNTF that connect the statistically dependent variables with the independent standard normal ones can thus be established in terms of Hermite polynomials:

$$
X_{i}=\mu_{x_{i}}+\sigma_{x_{i}} \sum_{k=0}^{3} b_{k i} H_{k}\left(\sum_{m=1}^{i} l_{i m} U_{m}\right),(i=1,2, \cdots, n)
$$

or in the form of classical formulation:

$$
X_{i}=\mu_{x_{i}}+\sigma_{x_{i}}\left[a_{0 i}+a_{1 i} \sum_{m=1}^{i} l_{i m} U_{m}+a_{2 i}\left(\sum_{m=1}^{i} l_{i m} U_{m}\right)^{2}+a_{3 i}\left(\sum_{m=1}^{i} l_{i m} U_{m}\right)^{3}\right],(i=1,2, \cdots, n)
$$

Using Eq. (41) or Eq. (42), normal transformation can then be directly performed for correlated nonnormal variables with their first four moments and correlation coefficients only, whereas the information on joint PDF/marginal PDFs is not required. The transformation expressed in Eq. (42) is an extension of the work reported in ( $\mathrm{Lu}$ et al., 2017), where the transformation is achieved based on the first three moments.

\section{Comparison and application of PNTF for structural reliability anal- ysis}

Although the PNTF was originally developed for the simulation of non-normal random variables, it can be further extended to aid structural reliability analysis in different ways. In this section, some existing attempts of using PNTF for structural reliability analysis are examined, and the newly derived Hermite PNTF is incorporated with conventional reliability analysis methods, e.g. MCS, FORM and SORM, to deal problems with correlated random variables. The third-order PNTF is exclusively adopted in the numerical study, while the observation and conclusion hold for more general cases.

\subsection{MCS using PNTF}

Since the PNTF was originally developed for simulating non-normal random variables, its application in MCS is straightforward. The MCS method for structural reliability analysis using second- and thirdorder PNTF was proposed by Zhao (Zhao et al., 2002), where the method was found simple to use and yet accurate enough to include the random variables with unknown CDF/PDF. In this section, the application of PNTF for reliability analysis using MCS will be illustrated for problems with independent random variables and correlated random variables, respectively. 
Table 1: Distribution information and statistical parameters of the random variables.

\begin{tabular}{lcccccccr}
\hline \multirow{2}{*}{ Distribution } & \multicolumn{4}{c}{ Statistical Moments } & \multicolumn{4}{c}{ Parameters } \\
\cline { 2 - 9 } & $\mu$ & $\sigma$ & $\alpha_{3}$ & $\alpha_{4}$ & $a_{0}$ & $a_{1}$ & $a_{2}$ & $a_{3}$ \\
\hline Lognormal & 120 & 24 & 0.6080 & 3.6644 & -0.09791 & 0.96964 & 0.09791 & 0.00686 \\
Gamma & 80 & 25 & 0.6250 & 3.5859 & -0.10326 & 0.98129 & 0.10326 & 0.00266 \\
Gumbel & 100 & 40 & 1.1396 & 5.4000 & -0.16827 & -0.89695 & 0.16827 & -0.02417 \\
Weibull & 120 & 48 & 0.2768 & 2.7875 & -0.05066 & 1.04205 & 0.05066 & -0.01510 \\
\hline
\end{tabular}

\subsubsection{Cases with independent random variables}

When all input random variables are independent, the basic steps of PNTF-based MCS for reliability analysis are listed below:

1. Initialize the number of sampling points $N$.

2. Collect the information of the first few moments, i.e. mean, standard deviation, skewness and kurtosis, of random variables, either using the PDF or the available samples of the variables.

3. Determine the polynomial coefficients using the first few moments of random variables by momentmatching method, e.g. solving Eqs. (4-7) to establish the PNTF for each random variable.

4. Generate a set of standard normal random variables for each non-normal random variable, and substitute them into their corresponding PNTF to generate samples with desired non-normal distributions or moment properties.

5. Substitute the samples of random variables generated in step 4 into the performance function $g(\mathbf{X})$ to determine the value of performance function for each sample.

6. Count the number of samples $N_{f}$ that fall in the failure domain, i.e. $g(\mathbf{X}) \leq 0$.

7. Estimate the failure probability as $P_{f}=\frac{N_{f}}{N}$.

8. Check the convergence condition: if met, stop calculation; otherwise, generate more samples and add into $N$, repeat from step 4 to step 7 until convergence criterion is reached.

Example 1: This example aims to investigate the accuracy and efficiency of the PNTF-based MCS for the simulation of some commonly used distributions. Four different distributions are considered, e.g. Lognormal, Gamma, Gumbel and Weibull, and the corresponding first four moments are listed in Table 1. With the given moments of the four distributions and solving the equations, e.g. Eqs. (4-7) or Eqs. (14-16), the corresponding coefficients for each distributions can be obtained, as recorded in Table 1. Once the polynomial coefficients are obtained, the random samples for each specific distribution can readily be generated using the third-order PNTF, i.e. Eq. (3). The histograms obtained using PNTFbased MCS with a sample size of 1,000,000 for the four distributions are shown in Fig. 4 along with the exact PDF indicated with the solid lines. For all four distributions, good agreement is observed between the histogram plotted from MCS and the exact PDF.

Example 2: In order to investigate the performance of the PNTF-based MCS for structural reliability analysis, this example considers the following non-linear performance function:

$$
G(\mathbf{X})=X_{3}-\sqrt{300 X_{1}^{2}+1.92 X_{2}^{2}}
$$




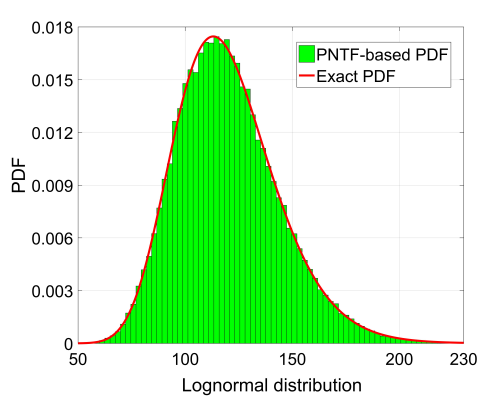

(a)

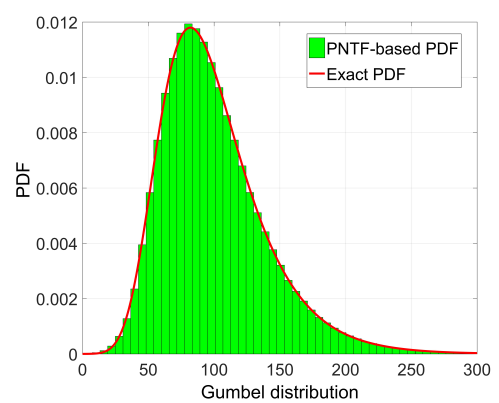

(c)

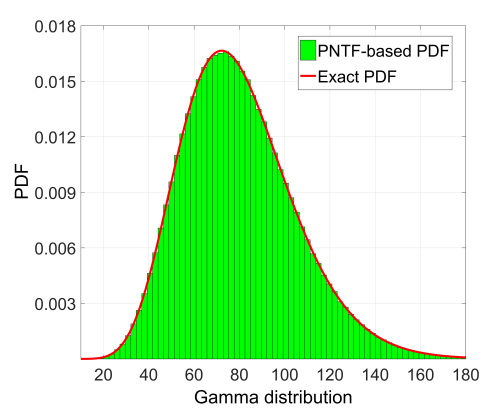

(b)

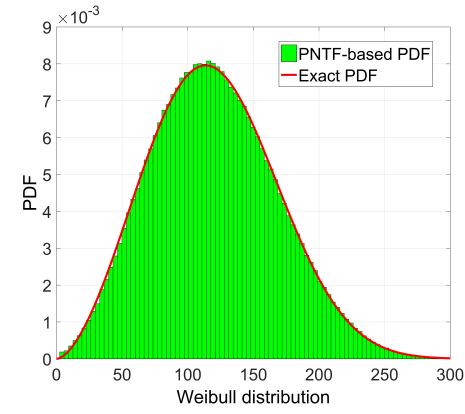

(d)

Figure 4: Comparison of the histogram generated using PNTF-based MCS and the exact PDF (a) Lognormal distribution (b) Gamma distribution (c) Gumbel distribution (d) Weibull ditribution.

Table 2: Distribution information and statistical parameters of the random variables

\begin{tabular}{lccccc}
\hline Random variables & Distribution & Mean & Standard deviation & Skweness & Kurtosis \\
\hline$X_{1}$ & Lognormal & 1.0 & 0.16 & 0.4841 & 3.4195 \\
$X_{2}$ & Gumbel & 20.0 & 2.0 & 1.1396 & 5.4000 \\
$X_{3}$ & Weibull & 48 & 3.00 & -0.8658 & 4.2596 \\
\hline
\end{tabular}

where $X_{1}, X_{2}, X_{3}$ are three independent random variables, whose distribution information and statistical parameters are listed in Table 2. With the given distribution information of the random variables, the failure probability can be readily calculated using the classical reliability analysis methods, e.g. FORM, SORM and MCS. The failure probability and the corresponding reliability indices obtained using FORM, SORM and MCS (with a sample size of 1,000,000) are recorded in Table 3 along with the exact results computed by direct integration.

Since the statistical moments, i.e. mean, standard deviation, skewness and kurtosis, are known for all input random variables, the failure probability can also be calculated following the PNTF-based MCS (with a sample size of 1,000,000), and the results are recorded in Table 3. It is observed that the results from the PNTF-based MCS agrees well with that obtained using conventional MCS with the information of $\mathrm{CDF} / \mathrm{PDF}$, and the relative errors are $0.17 \%$ and $0.06 \%$, respectively. This agreement in failure probability calculation is expected from the good match of the histogram generated using PNTF-based MCS and the exact PDF of the random variables, as shown in Fig. 5. On the other hand, however, the result provided by FORM exhibits large error, i.e. as high as $44.75 \%$. The reason is that the performance function is of non-linear form, the first-order Taylor series expansion in FORM cannot accurately capture the non-linearity of the limit state surface and thus results in a large error of the failure probability estimation. Since the curvatures of the limit state surface are considered in SORM, 
Table 3: Results for example 2 using different methods

\begin{tabular}{lccr}
\hline Methods & Reliability index $(\beta)$ & Failure probability $\left(P_{f}\right)$ & $e \%=\frac{\left|p_{f(\text { Exact })}-p_{f}\right|}{p_{f(\text { Exact })}} \times 100$ \\
\hline FORM & 3.0845 & $1.0195 \times 10^{-3}$ & 44.75 \\
SORM & 2.8960 & $1.8895 \times 10^{-3}$ & 2.41 \\
Conventional MCS & 2.9033 & $1.8462 \times 10^{-3}$ & 0.06 \\
PNTF-based MCS & 2.9030 & $1.8483 \times 10^{-3}$ & 0.17 \\
Exact & 2.9035 & $1.8451 \times 10^{-3}$ & $\sim$ \\
\hline
\end{tabular}
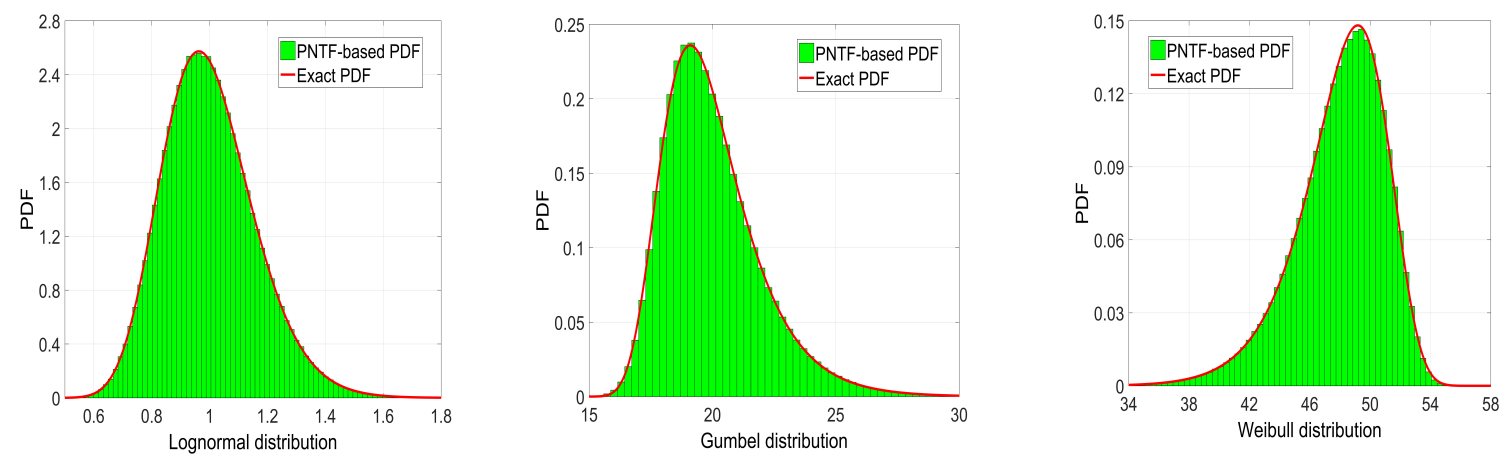

Figure 5: Comparison of the histogram generated using PNTF and the exact PDF for the variables.

a better approximation to the failure surface is expected and thus it offers a more accurate estimation of the failure probability.

Through this example, it is observed that an accurate estimation can be achieved using the PNTFbased MCS. The reliability analysis can be directly conducted using the PNTF-based MCS even when the $\mathrm{CDF} / \mathrm{PDF}$ of the random variables are unavailable, whereas only the statistical moments are known. This property makes it especially attractive when only samples of the random variables are available. However, it inherits the drawbacks of conventional MCS when dealing with small failure probabilities, especially for problems involving time-consuming numerical analysis such as finite element simulation. The application of PNTF in more advanced simulation methods such as importance sampling still needs further investigation.

\subsubsection{Cases with correlated random variables}

The implementation of PNTF-based MCS for reliability problems with independent random variables is quite straightforward by directly applying the PNTF for each variable. For cases with correlated random variables, however, the PNTF should be extended to account for the correlation coefficients between random variables. In this section, two different PNTF-based MCSs are presented, named as PNTFbased MCS 1 and PNTF-based MCS 2, respectively. The two MCS schemes use different methods to generate correlated random samples.

\section{- PNTF-based MCS 1}

Suppose $n$ correlated non-normal random variables are involved in a reliability problem. The polynomial normal transformations for $i$-th and $j$-th variables are:

$$
\frac{X_{i}-\mu_{x_{i}}}{\sigma_{x_{i}}}=X_{S i}=a_{0 i}+a_{1 i} Z_{i}+a_{2 i} Z_{i}^{2}+a_{3 i} Z_{i}^{3}
$$




$$
\frac{X_{j}-\mu_{x_{j}}}{\sigma_{x_{j}}}=X_{S j}=a_{0 j}+a_{1 j} Z_{j}+a_{2 j} Z_{j}^{2}+a_{3 j} Z_{j}^{3}
$$

where $X_{i}$ and $X_{j}$ are the desired correlated non-normal random variable with correlation coefficient of $\rho_{x_{i} x_{j}}, X_{S i}$ and $X_{S j}$ are the standardized variable of $X_{i}$ and $X_{j}$ with zero mean and unit variance, $Z_{i}$ and $Z_{j}$ are the standard normal random variable, which can be generated and correlated according to the following theorem (Headrick and Sawilowsky, 1999):

Theorem 1. Let $r_{i}$ be real-valued number inside the interval $[-1,1]$ for $i=0,1, \cdots, n$, and let $K_{1}, V, E_{1}, \cdots, E_{n} \sim$ i.i.d $N(0,1)$. Futher, let $K_{t+1}=r_{0} K_{1}+V \sqrt{1-r_{i}^{2}}$, where $t=1$ if $r_{0}<1$, and $t=0$ if $r_{0}=1$. If $Z_{i}=r_{i} K_{1}+E_{i} \sqrt{1-r_{i}^{2}}$ and $Z_{j}=r_{j} K_{t+1}+E_{j} \sqrt{1-r_{j}^{2}}$. Then, $Z_{i}, Z_{j} \sim N(0,1)$, and the correlation coefficient between $Z_{i}$ and $Z_{j}$ is: $\rho_{z_{i} z_{j}}=r_{0} r_{i} r_{j}$ when $t=1 ; \rho_{z_{i} z_{j}}=r_{i} r_{j}$ when $t=0$; In particular, $\rho_{z_{i} z_{j}}=r^{2}$ when $r_{i}=r_{j}=r$ and $t=0$.

It is referred to (Headrick and Sawilowsky, 1999) for the full proof of the above theorem. Following the definition of correlation coefficient and Theorem 1, a general equation that solves $\rho_{z_{i} z_{j}}$ for specified values of $\rho_{x_{i} x_{j}}$ can be derived as follows with the aid of parameters in Eqs. (44-45):

$$
\rho_{x_{i} x_{j}}=r_{0} r_{i} r_{j}\left(a_{1 i} a_{1 j}+3 a_{1 j} a_{3 i}+3 a_{1 i} a_{3 j}+9 a_{3 i} a_{3 j}+2 a_{0 i} a_{0 j} r_{0} r_{i} r+6 a_{3 i} a_{3 j} r_{0}^{2} r_{i}^{2} r_{j}^{2}\right)
$$

or more generally as:

$$
\rho_{x_{i} x_{j}}=\rho_{z_{i} z_{j}}\left(a_{1 i} a_{1 j}+3 a_{1 j} a_{3 i}+3 a_{1 i} a_{3 j}+9 a_{3 i} a_{3 j}+2 a_{0 i} a_{0 j} \rho_{z_{i} z_{j}}+6 a_{3 i} a_{3 j} \rho_{z_{i} z_{j}}^{2}\right)
$$

For any given $k$ non-normal variables that may be desired, in order to ensure simultaneous solutions for all pairwise intercorrelations in Eqs. (46-47), it is suggested to impose certain restrictions to Theorem 1 and is given as follows (Headrick and Sawilowsky, 1999):

1. If $k=2$, then $\rho_{z_{i} z_{j}}=r$ by restricting $r_{0}=1$ and $r_{i}=r_{j}=r$. This results in one equation of the form of Eq. (46) or Eq. (47) with one unknown, $r$.

2. If $k=3$, then $\rho_{z_{i} z_{j}}=r_{i} r_{j}$ by restricting $r_{0}=1$. This results in three equations of the form of Eq. (46) or Eq. (47) with three unknowns, $r_{1}, r_{2}$ and $r_{3}$.

3. If $k>3$, then $\rho_{z_{i} z_{j}}=r_{0} r_{i} r_{j}$ where $r_{0}=\prod_{i=1}^{s} t_{i}, s=k(k-3) / 2$. A system of $I=k(k-1) / 2$ equations of the form of Eq. (46) or Eq. (47) can be derived with a series of unknowns, e.g. $r_{1}$, $r_{2}, r_{3}, r_{4}, t_{1}$ and $t_{2}$ for $k=4$.

Thus, any two correlated normal random variables, $Z_{i}$ and $Z_{j}$, can be generated according to Theorem 1. Then, they can be substituted into Eqs. (44-45) to obtain $X_{i}$ and $X_{j}$ that are correlated with the desired correlation coefficients and possess the specified moments.

The basic procedure of PNTF-based MCS 1 for reliability analysis with correlated random variables are summarized asfollows:

1. Collect the information of the first few moments, i.e. mean, standard deviation, skewness and kurtosis, and correlation coefficients of random variables, either using the PDF or the available samples of the variables.

2. Determine the polynomial coefficients using the first few moments of random variables by momentmatching method, to establish the PNTF for each random variable.

3. Determine the values of $r_{i}, i=0,1, \cdots, n$ by substituting the desired values of $\rho_{x_{i} x_{j}}$ and polynomial parameters $\left(a_{0 i}, a_{1 i}, a_{2 i}, a_{3 i} ; a_{0 j}, a_{1 j}, a_{2 j}, a_{3 j}\right)$ obtained in step 2 into Eq. (46) and simultaneously solving for $r_{i}, i=0,1, \cdots, n$. Then, $\rho_{z_{i} z_{j}}=r_{0} r_{i} r_{j}$ can be obtained. 


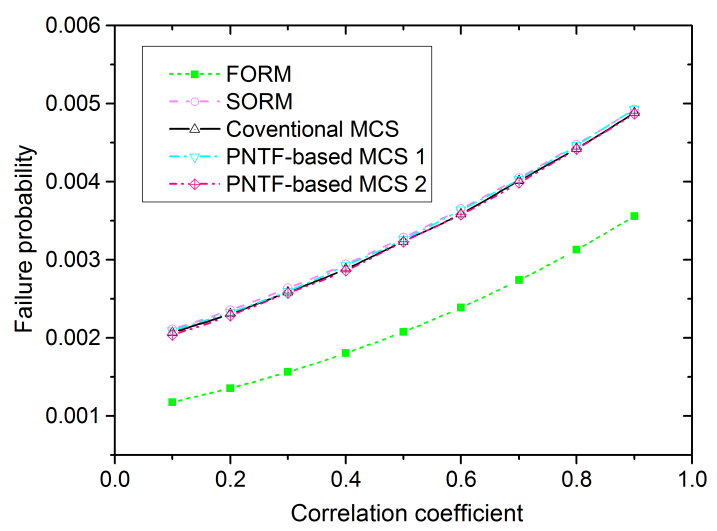

(a)

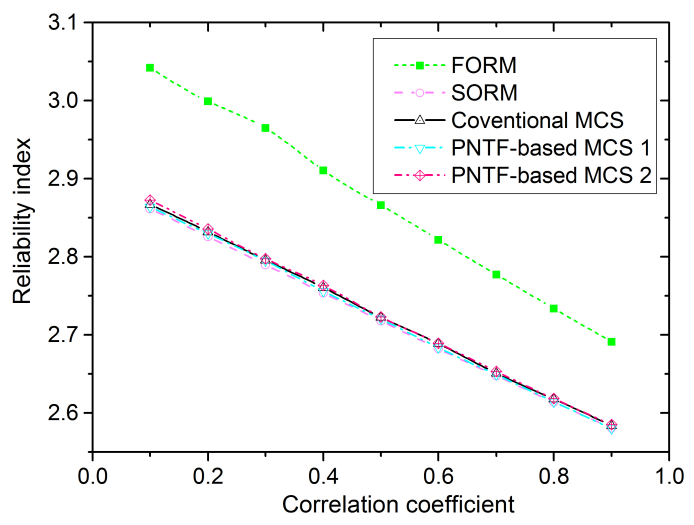

(b)

Figure 6: Results using different methods (a) Failure probability (b) Reliability index.

4. Substitute the values of $r_{i}, i=0,1, \cdots, n$ into the equations in Theorem 1 to generate samples of $Z_{i}, i=0,1, \cdots, n$ for each random variable.

5. Substitute $Z_{i}, i=0,1, \cdots, n$ from step 4 and their corresponding polynomial parameters obtained from step 2 into Eq. (44) to generate samples of specified distributions (or moment properties) with the desired correlation coefficients.

6. Substitute the samples of random variables generated in step 5 into the performance function $g(\mathbf{X})$ to determine the value of performance function for each sample vector.

7. Count the number of samples $N_{f}$ that fall in the failure domain, i.e. $g(\mathbf{X}) \leq 0$.

8. Estimate the failure probability as $P_{f}=\frac{N_{f}}{N}$.

9. Check the convergence condition: if met, stop calculation; otherwise, generate more samples, repeat from step 4 to step 8 until convergence criterion is reached.

\section{- PNTF-based MCS 2}

The PNTF presented in (Zhao and $\mathrm{Lu}, 2007$ ), i.e. Eq. (14), performs quite well for independent variables. For dependent random variables, however, this PNTF is unfeasible. In this section, Eq. (14) is extended to cope with correlated random variables with the aid of Eq. (42), where the parameter vector $\mathbf{a}=\left(a_{0 i}, a_{1 i}, a_{2 i}, a_{3 i}\right)$ is calculated using Eqs. (15-16).

The basic procedure of PNTF-based MCS 2 is similar to the PNTF-based MCS 1, and the only difference is that instead of using Theorem 1, the newly derived Eq. (42) is employed to generate correlated random variables with the desired correlation coefficients. Described below, Example 3 demonstrates the performance of these two PNTF-base MCSs for reliability analysis.

Example 3: In this example the same performance function and distribution parameters as in Example 2 are considered, but it is assumed that $X_{1}, X_{2}$ are correlated with correlation coefficient varies from $\rho_{x_{1} x_{2}}=0.1$ to $\rho_{x_{1} x_{2}}=0.9$. The results of failure probability and corresponding reliability indices using different methods are shown in Fig. 6.

It is observed from Fig. 6 that both SORM and the PNTF-based MCS methods can provide very accurate results compared with the conventional MCS for all investigated cases with different correlation coefficients. Since the performance function studied is of non-linear type, the results provided by FORM have large errors. It is also interesting to notice that the failure probability increases as the correlation coefficient grows, whereas the reliability index decreases. 
Table 4: Comparison between the original correlation coefficient $\rho_{x_{1} x_{2}}$ and the one obtained using PNTF-based $\operatorname{MCS} \rho_{x_{1} x_{2}}^{*}$

\begin{tabular}{ccccccccccc}
\hline & $\rho_{x_{1} x_{2}}$ & 0.1 & 0.2 & 0.3 & 0.4 & 0.5 & 0.6 & 0.7 & 0.8 & 0.9 \\
\hline \multirow{2}{*}{$\rho_{x_{1} x_{2}}^{*}$} & PNTF-based MCS 1 & 0.0993 & 0.1992 & 0.3022 & 0.3992 & 0.4997 & 0.6003 & 0.7004 & 0.8000 & 0.9002 \\
\cline { 2 - 10 } & PNTF-based MCS 2 & 0.1010 & 0.2001 & 0.2990 & 0.4014 & 0.5006 & 0.6001 & 0.7003 & 0.7997 & 0.9004 \\
\hline
\end{tabular}

Moreover, based on the samples generated using PNTF-based MCS 1 and PNTF-based MCS 2, the correlation coefficient between $X_{1}$ and $X_{2}$ can readily be calculated, which is given in Table 4 along with the original coefficient correlations. As is clearly shown in the table that the correlation coefficients provided by these two PNTF-based MCS methods match quite well with the original ones, which in a way justifies the accuracy of using the proposed PNTF-based MCS approaches to generate correlated random variables with the specified correlation coefficient.

\subsection{PNTF based FORM/SORM}

FORM and SORM are arguably the most widely used methods for the reliability analysis of linear and slightly non-linear problems. With the presence of non-normal random variables, normal transformation techniques such as Rosenblatt transformation and Nataf transformation are usually required in order to conduct the reliability analysis in the standard normal space. In practical engineering problems, the joint PDF and marginal PDFs are often unknown due to the lack of data and the statistical information is easier to be expressed in terms of statistical moments and correlation coefficients. In such cases, the conventional normal transformation techniques, such as Rosenblatt transformation and Nataf transformation, cannot be applied, unless additional effort is made to fit an approximate distribution function. The PNTF provides an alternative approach to conduct the normal transformation such that FORM and SORM can still be used to calculated the failure probability even when the CDF/PDF of random variables are unknown (Zhao and Ono, 2000; Zhao and Lu, 2007).

\subsubsection{Cases with independent random variables}

In this section, the third-order PNTF is used to illustrate the basic procedure of PNTF-based FORM/SORM for reliability analysis with independent random variables. The inverse of the PNTF in Eq. (14) is required for the PNTF-based FORM/SORM, which is given as (Zhao and Lu, 2007):

$$
\begin{gathered}
u=-\frac{\sqrt[3]{2} p}{\sqrt[3]{-q+\Delta}}+\frac{\sqrt[3]{-q+\Delta}}{\sqrt[3]{2}}-\frac{l_{1}}{3 k_{2}} \\
\Delta=\sqrt{q^{2}+4 p^{3}} \\
p=\frac{3 k_{1} k_{2}-l_{1}^{2}}{9 k_{2}^{2}} \\
q=\frac{2 l_{1}^{3}-9 k_{1} k_{2} l_{1}+27 k_{2}^{2}\left(-l_{1}-x_{S}\right)}{27 k_{2}^{3}}
\end{gathered}
$$

Based on the third-order PNTF in Eq. (14), the Jacobian matrix of the transformation corresponding to a random variable with unknown $\mathrm{CDF} / \mathrm{PDF}$ can be derived as:

$$
J_{i i}=\frac{\partial x_{i}}{\partial z_{i}}=\sigma_{x_{i}}\left[k_{1}+2 l_{1} z_{i}+3 k_{2} z_{i}^{2}\right]
$$

The computational procedure of the PNTF-based FORM/SORM with independent random variables is similar to the conventional FORM/SORM, and the only difference is that the normal transformation and its inverse are conducted using Eq. (14) and Eq. (48), respectively, and the Jacobian matrix is calculated using Eq. (52). The main steps are: 
Table 5: Distribution information and statistical parameters of the random variables

\begin{tabular}{lccccr}
\hline Random variables & Distribution & Mean & C.O.V & Skweness & Kurtosis \\
\hline$X_{1}$ & Lognormal & 0.6 & 0.131 & 0.3953 & 3.2790 \\
$X_{2}$ & Gumbel & 2.18 & 0.03 & 1.1396 & 5.4000 \\
$X_{3}$ & Weibull & 32.8 & 0.03 & -1.0041 & 4.7905 \\
\hline
\end{tabular}

1. Collect the information of the first few moments, e.g. mean, standard deviation, skewness and kurtosis, and correlation coefficients of random variables, either using the PDF or the available samples of the variables.

2. Determine the polynomial coefficients using the first few moments of random variables by momentmatching method, e.g. solving Eqs. (15-16) or Eq. (27), to establish the PNTF for each random variable.

3. Transform the performance function $G(\mathbf{X})$ with independent non-normal random variables into a performance function $G(\mathbf{U})$ of independent standard normal random variables using Eq. (14).

4. Compute the reliability index and failure probability using the classical FORM / SORM procedure. When calculating the derivative of performance function with respect to the original variables X, the element of Jacobian matrix of normal transformation for variables with known CDF/PDF can be calculated using Rosenblatt transformation, Nataf transformation or Eq. (52), whereas for variables with unknown $\mathrm{CDF} / \mathrm{PDF}$ only Eq. (52) can be used to calculate the element of Jacobian matrix.

Described below, Example 4 demonstrates the applicability and accuracy of PNTF-based FORM/SORM in structural reliability analysis.

Example 4: This example considers a non-linear performance function,

$$
G(X)=567 f r-0.5 H^{2}
$$

and the statistical parameters of these three input random variables are given in Table 5 .

Since the distributions of random variables are known, the failure probability can be readily calculated using the classical FORM, SORM and MCS. The results obtained using FORM, SORM and MCS (with a sample size of 1000,000) are listed in Table 6. For comparison purpose, assuming the distributions for input random variables are unknown and the only statistical information available are the first four moments. In this case, the normal transformation and its inverse are conducted using Eq. (14) and Eq. (48), respectively, and the Jacobian matrix is calculated using Eq. (52). The results of the PNTF-based FORM/SORM are recorded in Table 6 along with the results for PNTF-based MCS.

Shown in Table 6, the PNTF-based MCS can obtain almost the same result as the reference one obtained using conventional MCS with a sample size of 1,000,000. Both the conventional FORM/SORM and the PNTF-based FORM/SORM can provide a failure probability estimation with the same level of accuracy as their respective counterparts. However, since the performance function is of highly nonlinearity, both the conventional FORM and the PNTF-based FORM produce large errors for the failure probability estimation; whereas both the conventional SORM and the PNTF-based SORM can improve the results significantly by taking into consideration the curvature of the limit state surface.

\subsubsection{Cases with correlated random variables}

The PNTF-based FORM/SORM as illustrated in the previous section can offer comparable accuracy as the classical FORM/SORM. However, the aforementioned PNTF-based FORM/SORM can only be 
Table 6: Results for example 4 using different methods

\begin{tabular}{cccc}
\hline Methods & Reliability index $(\beta)$ & Failure probability $\left(P_{f}\right)$ & $e \%=\frac{\left|p_{f(\text { Exact })}-p_{f}\right|}{p_{f(\text { Exact })}} \times 100$ \\
\hline FORM & 2.1092 & 0.01747 & 18.20 \\
SORM & 2.1652 & 0.01519 & 2.77 \\
PNTF-based FORM & 2.1051 & 0.01764 & 19.35 \\
PNTF-based SORM & 2.1648 & 0.01520 & 2.84 \\
PNTF-based MCS & 2.1756 & 0.01479 & 0.07 \\
Conventional MCS & 2.1759 & 0.01478 & $\sim$ \\
\hline
\end{tabular}

applied to problems with independent random variables. In this section, the extension to cope with correlated random variables is examined.

Based on the theory presented in Section 3.2 and Section 4.1.2, the substitution of Eq. (41) or Eq. (42) into the performance function transforms the performance function of correlated random variables into a function of independent standard normal variables, and the reliability analysis methods such as FORM/SORM can thus easily be performed. The Jacobian matrix $\mathbf{J}_{\mathbf{X U}}$ of the transformation corresponding to a random vector with unknown $\mathrm{CDF} / \mathrm{PDF}$ can be derived as:

$$
\mathbf{J}_{\mathbf{X U}}=\mathbf{L}^{\mathbf{T}} \mathbf{J}_{\mathbf{X Z}}
$$

where the matrix $\mathbf{L}$ is given in Eq. (38) and the elements of $\mathbf{J}_{\mathbf{X Z}}$ can be calculated using the following equation:

$$
J_{x_{i} z_{i}}=\frac{\partial x_{i}}{\partial z_{i}}=\sigma_{x_{i}}\left[k_{1}+2 l_{1} z_{i}+3 k_{2} z_{i}^{2}\right]
$$

The computational procedure of the PNTF-based FORM/SORM with dependent random variables is similar to the conventional FORM/SORM, and the only difference is that for random variables with unknown $\mathrm{CDF} / \mathrm{PDF}$, the normal transformation and the Jacobian matrix are calculated using Eq. (42) and Eq. (54), respectively. Described below, three examples, two with known PDFs and one with unknown PDFs, demonstrate the applicability of the PNTF-based FORM/SORM for reliability analysis.

\section{- Correlated random variable with known PDF}

Example 5: In this example, the PNTF-based FORM/SORM is applied to calculate the failure probability for the performance function given in Example 2, where random variables $X_{1}, X_{2}$ are assumed correlated with the correlation coefficient varies from $\rho_{x_{1} x_{2}}=0.1$ to $\rho_{x_{1} x_{2}}=0.9$. The results of failure probability and reliability index using different methods are shown in Fig. 7. The PNTF-based FORM and SORM can both provide results with comparable accuracy as those given by FORM and SORM using Nataf transformation. Since the performance function is non-linear, only the PNTF-based SORM and SORM using Nataf transformation can provide a good estimate of failure probability as compared with that of MCS over the whole investigation range, whereas the PNTF-based FORM and the Natafbased FORM produce large errors.

In order to further investigate the applicability and accuracy of the PNTF-based FORM/SORM, it is important to study the performance of normal transformation embedded. Table 7 lists the correlation coefficients $\rho_{z_{i} z_{j}}$ in the standard normal space obtained using PNTF along with those obtained by Nataf transformation. Almost identical correlation coefficients in the standard normal space can be obtained using PNTF and Nataf transformation for all investigated cases, which further verify the accuracy of PNTF.

Example 6: A frame structure as shown in Fig. 8a is studied in this example. The statistical information of the member strength and load parameters are listed in Table 8. According to the study 


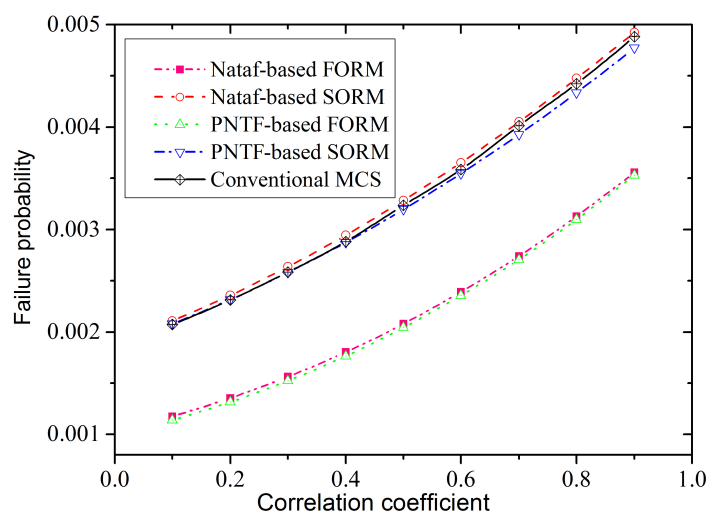

(a)

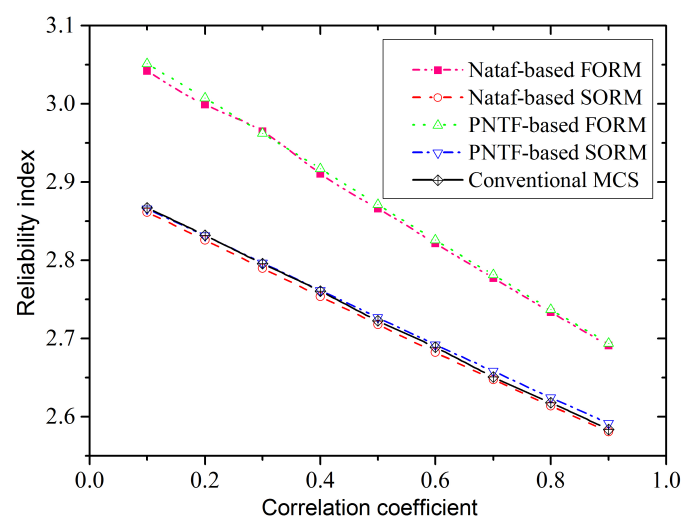

(b)

Figure 7: Results using different methods (a) Failure probability (b) Reliability index.

Table 7: Correlation coefficient in the standard normal space obtained using different approaches

\begin{tabular}{ccccccccccc}
\hline$\rho_{x_{1} x_{2}}$ & 0.1 & 0.2 & 0.3 & 0.4 & 0.5 & 0.6 & 0.7 & 0.8 & 0.9 \\
\hline$\rho_{z_{1} z_{2}}$ & PNTT & 0.1035 & 0.2064 & 0.3088 & 0.4105 & 0.5117 & 0.6123 & 0.7124 & 0.8119 & 0.9109 \\
\cline { 2 - 10 } & Nataf & 0.1034 & 0.2063 & 0.3085 & 0.4103 & 0.5115 & 0.6122 & 0.7125 & 0.8123 & 0.9117 \\
\hline
\end{tabular}

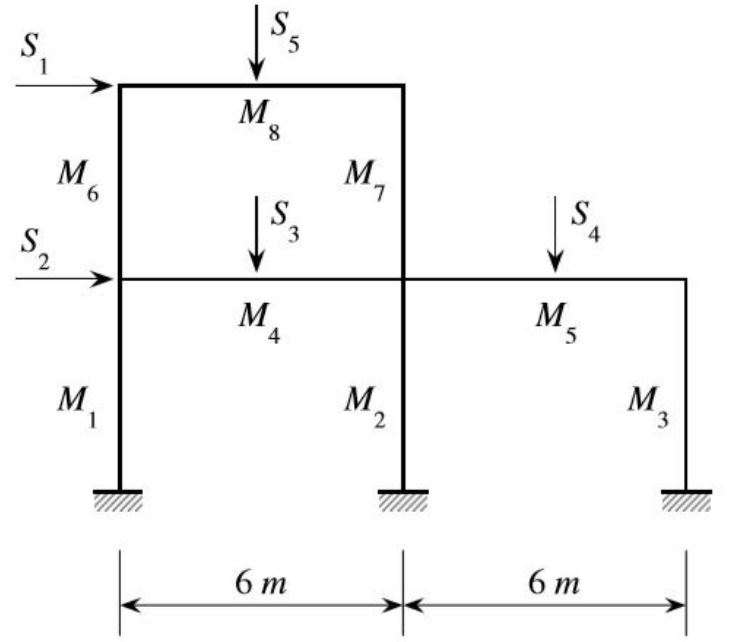

(a)
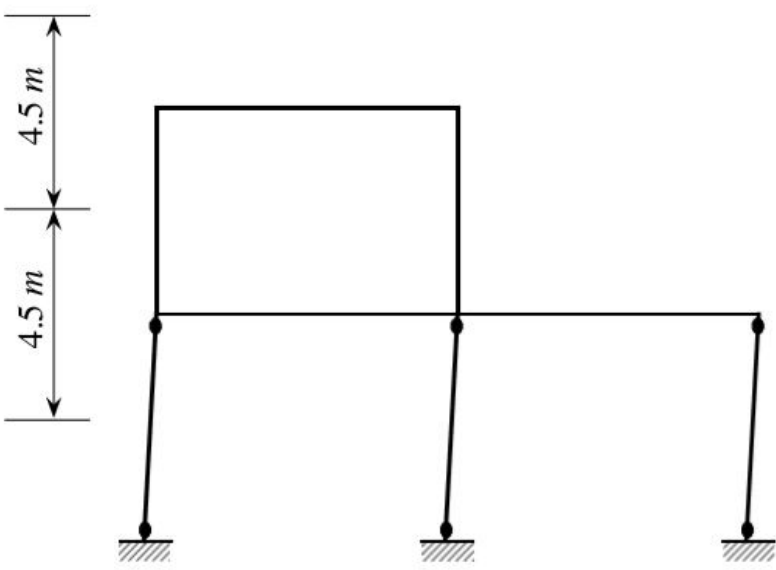

(b)

Figure 8: Structural illustration for Example 6 (a) Two-story two-bay frame structure and (b) Most likely failure mode (after (Lu et al., 2017)). 
Table 8: Distribution information and statistical parameters of the random variables

\begin{tabular}{cccccc}
\hline Random variables & Distribution & Mean & Standard deviation & Skweness & Kurtosis \\
\hline$M_{1}, M_{2}, M_{3}(\mathrm{kN} \cdot \mathrm{m})$ & Lognormal & 700 & 105 & 0.4534 & 3.3677 \\
$M_{6}, M_{7}(\mathrm{kN} \cdot \mathrm{m})$ & Lognormal & 700 & 105 & 0.4534 & 3.3677 \\
$M_{4}(\mathrm{kN} \cdot \mathrm{m})$ & Lognormal & 1500 & 225 & 0.4534 & 3.3677 \\
$M_{5}(\mathrm{kN} \cdot \mathrm{m})$ & Lognormal & 1200 & 180 & 0.4534 & 3.3677 \\
$M_{8}(\mathrm{kN} \cdot \mathrm{m})$ & Lognormal & 90 & 13.5 & 0.4534 & 3.3677 \\
$S_{1}(\mathrm{kN})$ & Lognormal & 100 & 25 & 0.7656 & 4.0601 \\
$S_{2}(\mathrm{kN})$ & Lognormal & 200 & 50 & 0.7656 & 4.0601 \\
$S_{3}(\mathrm{kN})$ & Lognormal & 265 & 66.25 & 0.7656 & 4.0601 \\
$S_{4}(\mathrm{kN})$ & Lognormal & 180 & 45 & 0.7656 & 4.0601 \\
$S_{5}(\mathrm{kN})$ & Lognormal & 140 & 35 & 0.7656 & 4.0601 \\
\hline
\end{tabular}

conducted in (Zhao and Ono, 1998; Lu et al., 2017), the most likely failure mode of this frame is illustrated in Fig. 8b, and the limit state function is given as:

$$
G(\mathbf{X})=2 M_{1}+2 M_{2}+2 M_{3}-4.5 S_{1}-4.5 S_{2}
$$

Given the limit state function in Eq. (56), it is assumed that the member strength parameters $M_{i}(i=1,2,3)$ of the frame structure are independent of the load parameters $S_{i}(i=1,2)$ and the dependence between different random parameters are assembled in a correlation matrix as:

$$
C_{X}=\left(\begin{array}{ccccc}
1 & 0.21 & 0.21 & 0 & 0 \\
0.21 & 1 & 0.21 & 0 & 0 \\
0.21 & 0.21 & 1 & 0 & 0 \\
0 & 0 & 0 & 1 & 0.35 \\
0 & 0 & 0 & 0.35 & 1
\end{array}\right)
$$

The "exact" failure probability obtained using MCS with 80,000,000 samples is 3.8750e-7 and the corresponding reliability index is 4.9416 . With the given information of marginal distribution and correlation matrix, the classical Nataf-based FORM/SORM can be implemented without any difficulty, providing the reliability index estimation with a value of 4.9299 and 4.9204 , respectively. Based on the first four moments of the random variables, the reliability analysis can also be performed using the PNTF-based FORM/SORM as described above, and the reliability index is respectively obtained as 4.9658 and 4.9616 , which are similar with the results from by their classical counterparts and all in close agreement with that of MCS.

\section{- Correlated random variable with unknown PDF}

Example 7: In this example an H-shaped steel column studied in (Zhao and Lu, 2007) (for independent variables) is considered, and the performance function is expressed as:

$$
G(X)=A Y-C
$$


Table 9: Statistical moments of the random variables

\begin{tabular}{ccccc}
\hline \multirow{2}{*}{ Variable } & \multicolumn{4}{c}{ Statistical Parameters } \\
\cline { 2 - 5 } & Mean & Standard deviation & Skewness & Kurtosis \\
\hline$A\left(\mathrm{~cm}^{2}\right)$ & 71.656 & 3.691 & 0.709 & 3.692 \\
$Y\left(t / \mathrm{cm}^{2}\right)$ & 3.055 & 0.364 & 0.512 & 3.957 \\
$C(t)$ & 100 & 40 & 1.264 & 5.969 \\
\hline
\end{tabular}

Table 10: Results for example 7 using different methods

\begin{tabular}{|c|c|c|c|c|c|c|}
\hline Methods & Reliability index $(\beta)$ & Failure probability $\left(P_{f}\right)$ & \multicolumn{4}{|c|}{ Correlation matrix } \\
\hline PNTF-based FORM & 2.0180 & 0.02180 & \multicolumn{4}{|c|}{$\sim$} \\
\hline \multirow[t]{2}{*}{ PNTF-based SORM } & 2.0170 & 0.02185 & & & $\sim$ & \\
\hline & & & & 1 & -0.3099 & 0.5707 \\
\hline \multirow[t]{3}{*}{ PNTF-based MCS1 } & 2.0003 & 0.02273 & $C_{X}=$ & -0.3099 & 1 & -0.4383 \\
\hline & & & & 0.5707 & -0.4383 & 1 \\
\hline & & & & 1 & -0.3107 & 0.5699 \\
\hline \multirow[t]{2}{*}{ PNTF-based MCS2 } & 2.0015 & 0.02267 & $C_{X}=$ & -0.3107 & 1 & -0.4403 \\
\hline & & & & 0.5699 & -0.4403 & 1 \\
\hline
\end{tabular}

The statistical information of the random variables are listed in Table 9 and the correlation matrix is assumed as:

$$
C_{X}=\left[\begin{array}{ccc}
1 & -0.31 & 0.57 \\
-0.31 & 1 & -0.44 \\
0.57 & -0.44 & 1
\end{array}\right]
$$

Since only the statistical moments of the random variables are known in this example, the classical FORM/SORM cannot be directly used unless a proper distribution is identified for each random variable. Using the PNTF-based FORM/SORM, the failure probability estimation can be directly evaluated, and with the results are listed in Table 10 along with the results provided by the PNTF-based MCSs described in section 4.1.2. Based on the samples generated using PNTF-based MCS 1 and PNTF-based MCS 2, the correlation coefficients between random variables in the original space can also be obtained and given in Table 10 for comparison. The results obtained using PNTF-based FORM/SORM are almost the same and both give very good approximation to the results provided by the PNTF-based MCS. Moreover, the correlation matrices obtained by PNTF-based MCS 1 and PNTF-based MCS 2 are very close to the original correlation matrix of the random variables in this example.

\section{Conclusions}

The polynomial normal transformation method is presented for structural reliability analysis, to transform between non-normal and normal random variables. It requires only the first four moments and correlation matrix of the input random variables to work, which is especially attractive when only samples of the random variables are available. The PNTF is expressed in terms of Hermite polynomials and being extended to correlated cases. The following conclusions can be drawn from the comprehensive numerical studies: 
1. The Hermite polynomial normal transformation is equivalent to the classical power series formulation, but it is much easier to establish the relationship between the correlation coefficient in the original space and the normal space. In addition, when the PNTF is applied for random variables with known PDF, the use of Hermite polynomials can greatly simplify the coefficient calculation.

2. Using the PNTF-based MCS, samples of random variables with or without $\mathrm{CDF} / \mathrm{PDF}$ can be easily generated with sufficient accuracy both for independent and correlated random variables.

3. The newly derived equation for normal transformation, i.e. Eq. (42), is easy to implement and the polynomial normal transformation can provide good results for correlation coefficient in the standard normal space as compared with that of Nataf transformation.

4. The PNTF-based FORM/SORM utilizing the first few moments and correlation matrix of the random variables can provide comparable results as those given by classical FORM/SORM based on Nataf transformation, which complements existing methods for reliability analysis when the $\mathrm{CDF} / \mathrm{PDF}$ of the random variables are unknown.

However, it is noteworthy that the PNTF-based methods are accurate enough only in the applicable range of their corresponding PNTF, large errors may be introduced for problems with statistical moments outside the applicable range. In this case, the complete formula recently proposed in (Zhao et al., 2018a) might be an option to bypass the limitation of the present work, which is an aspect worth for further investigation.

\section{Acknowledgements}

The authors would like to thank the support from the China Scholarship Council, Swansea University and the Great Britain-China Educational Trust.

\section{References}

Aldosary, M., Wang, J., and Li, C. (2018). Structural reliability and stochastic finite element methods: State-of-the-art review and evidence-based comparison. Engineering Computations, 35(6):2165-2214.

Andrews, G., Askey, R., and Roy, R. (1999). Special Functions. Encyclopedia of Mathematics and its Applications. Cambridge University Press.

Au, S. and Beck, J. (2003). Important sampling in high dimensions. Structural safety, 25(2):139-163.

Au, S.-K. and Beck, J. L. (2001). Estimation of small failure probabilities in high dimensions by subset simulation. Probabilistic engineering mechanics, 16(4):263-277.

Breitung, K. (1984). Asymptotic approximations for multinormal integrals. Journal of Engineering Mechanics, 110(3):357-366.

Buet-Golfouse, F. (2015). A multinomial theorem for hermite polynomials and financial applications. Applied Mathematics, 6(06):1017.

Chen, X. and Tung, Y.-K. (2003). Investigation of polynomial normal transform. Structural Safety, $25(4): 423-445$.

Der Kiureghian, A., Lin, H.-Z., and Hwang, S.-J. (1987). Second-order reliability approximations. Journal of Engineering mechanics, 113(8):1208-1225.

Der Kiureghian, A. and Liu, P.-L. (1986). Structural reliability under incomplete probability information. Journal of Engineering Mechanics, 112(1):85-104. 
Fleishman, A. I. (1978). A method for simulating non-normal distributions. Psychometrika, 43(4):521532.

Hasofer, A. M. and Lind, N. C. (1974). Exact and invariant second-moment code format. Journal of the Engineering Mechanics division, 100(1):111-121.

Headrick, T. C. (2002). Fast fifth-order polynomial transforms for generating univariate and multivariate nonnormal distributions. Computational Statistics 83 Data Analysis, 40(4):685-711.

Headrick, T. C. (2011). A characterization of power method transformations through l-moments. Journal of Probability and Statistics, 2011.

Headrick, T. C. and Kowalchuk, R. K. (2007). The power method transformation: Its probability density function, distribution function, and its further use for fitting data. Journal of Statistical Computation and Simulation, 77(3):229-249.

Headrick, T. C. and Sawilowsky, S. S. (1999). Simulating correlated multivariate nonnormal distributions: Extending the fleishman power method. Psychometrika, 64(1):25-35.

Hohenbichler, M. and Rackwitz, R. (1981). Non-normal dependent vectors in structural safety. Journal of the Engineering Mechanics Division, 107(6):1227-1238.

Hu, C., Youn, B. D., Wang, P., et al. (2019). Engineering Design Under Uncertainty and Health Prognostics. Springer.

Lu, Z.-H., Cai, C.-H., and Zhao, Y.-G. (2017). Structural reliability analysis including correlated random variables based on third-moment transformation. Journal of Structural Engineering, 143(8):04017067.

Naess, A., Leira, B., and Batsevych, O. (2012). Reliability analysis of large structural systems. Probabilistic Engineering Mechanics, 28:164-168.

Nie, J. and Ellingwood, B. R. (2000). Directional methods for structural reliability analysis. Structural Safety, 22(3):233-249.

Rosenblatt, M. (1952). Remarks on a multivariate transformation. The annals of mathematical statistics, 23(3):470-472.

Shinozuka, M. (1983). Basic analysis of structural safety. Journal of Structural Engineering, 109(3):721740 .

Stuart, A., Arnold, S., Ord, J. K., O'Hagan, A., and Forster, J. (1994). Kendall's advanced theory of statistics. University Press.

Tadikamalla, P. R. (1980). On simulating non-normal distributions. Psychometrika, 45(2):273-279.

Tung, Y.-K., You, L., and Yoo, C. (2019). Third-order polynomial normal transform applied to multivariate hydrologic extremes. Water, 11(3):490.

Vale, C. D. and Maurelli, V. A. (1983). Simulating multivariate nonnormal distributions. Psychometrika, $48(3): 465-471$.

Winterstein, S. and Bjerager, P. (1987). The use of higher moments in reliability estimation. In Proc. ICASP 5, Int. Conf. on Appl. of Statistics and Prob. In Soil and Struct, volume 2, pages 1027-1036.

Xiao, Q. (2015). Generating correlated random vector by polynomial normal transformation. arXiv preprint arXiv:1508.06433.

Xiao, Q. (2017). Generating correlated random vector involving discrete variables. Communications in Statistics-Theory and Methods, 46(4):1594-1605. 
Xiu, D. and Karniadakis, G. E. (2002). The wiener-askey polynomial chaos for stochastic differential equations. SIAM journal on scientific computing, 24(2):619-644.

Yangtian Li, H. L. G. W. (2020). Dimension-adaptive algorithm based pce fo rmodels with many model parameters. Engineering Computations, 37(2):522-545.

Zhang, W. and Xiao, Y. (2019). An adaptive order response surface method for structural reliability analysis. Engineering Computations, 36(5):1626-1655.

Zhang, X.-Y., Zhao, Y.-G., and Lu, Z.-H. (2019). Straightforward hermite polynomial model with application to marine structures. Marine Structures, 65:362-375.

Zhao, Y.-G. and Lu, Z.-H. (2007). Fourth-moment standardization for structural reliability assessment. Journal of Structural Engineering, 133(7):916-924.

Zhao, Y.-G. and Ono, T. (1998). System reliability evaluation of ductile frame structures. Journal of Structural Engineering, 124(6):678-685.

Zhao, Y.-G. and Ono, T. (2000). Third-moment standardization for structural reliability analysis. Journal of Structural Engineering, 126(6):724-732.

Zhao, Y.-G. and Ono, T. (2001). Moment methods for structural reliability. Structural safety, 23(1):4775 .

Zhao, Y.-G., Ono, T., and Ishii, K. (2002). Monte carlo simulation using moments of random variables. Journal of Asian Architecture and Building Engineering, 1(1):13-20.

Zhao, Y.-G., Zhang, L.-W., Lu, Z.-H., and He, J. (2019). First passage probability assessment of stationary non-gaussian process using the third-order polynomial transformation. Advances in Structural Engineering, 22(1):187-201.

Zhao, Y.-G., Zhang, X.-Y., and Lu, Z.-H. (2018a). Complete monotonic expression of the fourth-moment normal transformation for structural reliability. Computers \& Structures, 196:186-199.

Zhao, Y.-G., Zhang, X.-Y., and Lu, Z.-H. (2018b). A flexible distribution and its application in reliability engineering. Reliability Engineering 8 System Safety, 176:1-12.

Zou, B. and Xiao, Q. (2014). Solving probabilistic optimal power flow problem using quasi monte carlo method and ninth-order polynomial normal transformation. IEEE Transactions on Power Systems, 29(1):300-306. 